\title{
Creating Realistic Three-Dimensional Human Shape Characters for Computer-Generated Films
}

\author{
Arghyro Paouri, Nadia Magnenat Thalmann, Daniel Thalmann
}

\begin{abstract}
In this paper, we discuss both the artistic and technical concerns involved in the construction of a computergenerated realistic human character. We investigate various methods for creating three-dimensional human shapes based on our experiences. The traditional approach consists in constructing the shapes from real human characters or plaster models. In the first part, we survey several methods that we have already used in the past: 3D reconstruction from 2D photos, 3D digitizing using a Polhemus digitizer, cross sections method and local deformations. Then, we show how a direct sculpting approach based on threedimensional devices like the spaceball or the dataglove could considerably improve the situation. We have obtained a realistic human character with a method similar to the modelling of clay, work which essentially consists of adding or eliminating parts of the material, and turning around the object when the principal form has been set up.
\end{abstract}

\section{INTRODUCTION}

Through our understanding of modern science and technology, we are able to use the computer to simulate realistic environments, including human characters with animation.

The synthesis of realistic characters leads to obtain and include the specific features of the character of interest. For the universally known personalities (actors) such as Marilyn, Humphrey, and Elvis, there is a less scope to make mistakes as the deviations will be very easily detected by the spectator. In spite of this ambition to make realism, or better, imitation, this type of realism should not be confused with the photographic or the cinematographic realism.

Creating a human body for an actor is only the first step, his particular character depends on his body movements and his personality is defined by the subtle changes of his facial expressions and other gestures.

\section{THE PROBLEM OF MODELING HUMAN SHAPES USING POLYGONAL MESHES}

From a geometric modelling point-of-view, the most popular technique is still the polygonal mesh, because most of the latest superworkstations provide facilities to process them by hardware (fast rendering and matrix operations). Although often expensive in terms of CPU time, polygonal models of 3D objects are the most common ones. One of the reasons for using this kind of model is that the latest superworkstations provide facilities to process them by hardware (fast rendering and matrix operations). In these models, all objects are decomposed into polygonal faces. For objects such as cubes or regular polyhedra, this decomposition is very effective. But for objects such as spheres or revolution surfaces, approximations are required. Unfortunately, even if revolution surfaces are simple shapes, large numbers of polygons are often needed. Also, the essential character of some surfaces (e.g. spheres) is lost when they are approximated by collections of polygons.

A surface like a human face is irregular and composed of bossels and depressions. It is important to choose vertices on the top of bossels and the bottom of depressions. Important angle variations between adjacent facets should be avoided, because they cause undesirable variations in the shading, a physical phenomenon known as the Mach effect. The only solution consists of increasing the number of facets in regions where the curvature is important. 
At this stage, the designer should be concerned with the animation:

1. First, the camera location must be considered: any curve (sequence of edges) which is shown from side-face must have numerous vertices. The more the camera eye near the vertices, the more the number of vertices should be increased, to make the surface smoother.

2. The actor motion also enters in consideration. A curve (sequence of edges) may vary in the animation. In this case the number of vertices should be increased for the maximum of curvature, to avoid any discontinuity of the surface during the motion.

\section{METHODS USING A REAL HUMAN MODEL OR A PLASTER MODEL}

Traditional methods to create irregular shapes like human shapes are based on digitizing. Three popular methods have been extensively used:

\section{Three-dimensional reconstruction from two-dimensional photographs}

Two or three projections (photos) are entered and the computer is used to derive the 3D coordinates. A photo of an object provides a perspective view of this object; the further the camera is from the object, the more parallel the view. Assume two parallel views at the same height; a fixed point will appear in two different locations. For example, consider a point on the shape to be digitized for a front view and a view at $60^{\circ}$. It is possible for the computer to determine the $3 \mathrm{D}$ location of the point from the two angles $\left(0^{\circ}\right.$ and $\left.60^{\circ}\right)$ and the $2 \mathrm{D}$ location of the point on both views. The model to be digitized should be placed onto a turntable; this allows the object to turn and the camera to be made immovable. Photo angles should be chosen so that each point of the object is on at least two photos. It is now necessary to number facets and vertices on each photo. Once the photos are ready, we used our three-dimensional digitizing and reconstruction program DIGIT3D (Magnenat Thalmann and Thalmann 1990). With DIGIT3D, each vertex should be present on two photos. Once each vertex has been entered using two photos, the digitizing procedure is terminated and a file of three-dimensional vertices is created. The last stage consists of establishing a text file containing the list of vertex numbers for each facet. Finally, the complete object may be generated using the file of vertices and the file of facets. Synthetic Marilyn and Bogey in the film Rendez-vous in Montreal (Magnenat Thalmann and Thalmann 1987) were created using this approach. Plate 1 shows a ray-traced Marilyn's image.

\section{Reconstruction from cross sections}

This popular method consists of reconstructing an object from a set of serial cross sections, like tracing the contours from a topographic map. This method (Magnenat Thalmann and Thalmann 1990) has been used to create Eglantine, a computerized mannequin, who never existed before.

\section{Three-dimensional digitizing}

The technique is simply to enter the 3D coordinates using a 3D digitizer. Three types of devices are now available: devices based on three orthogonal magnetic fields transmitted to a wand, devices based on three sound captors, devices based on laser light. We used for example, the Polhemus 3D-digitizer (based on magnetic fields) to create various objects. The method is less time-consuming than the two other methods, because no photos are needed. However, there are limitations in the shapes, which can be digitized; cavities and small parts cannot be entered.

To indicate to the computer the character shape in the three methods, we need the real person herself or a reduced model; otherwise a plaster model similar to the person should be created. For the reconstitution of dead actors, it is necessary to find a person who has about the same dimensions or to create a plaster model from photos.

But, what to enter into the computer ? For the reconstruction from cross-sections, the cross sections have to be digitized. For the two other methods, it is necessary to digitize (in 2D or in 3D) a polygonal mesh as discussed in the first section. If a plaster model has to be digitized, it should be large enough to allow the drawing of facets and vertices on it. However, if photos have to be taken, the plaster model should be small enough to be easily photographed. Any material may be chosen for the object; however, the surface should be of a light color and non-reflective, to allow the drawing of lines and photos if necessary

Plaster models have to be created in order to be similar to the real persons to be displayed. To do that, photos of the persons have to be found at a certain age. The methodology generally differs according to the 
part of the person. For the heads, the teeths, the hands, the arms and the fingers, a sculptor creates the plasters from photos of the real persons. For the Marilyn's body, a person similar to Marilyn for the dimensions has to be found. Plaster is then flown onto the person body to create a plaster body.

Instead of drawing the polygonal mesh on the plaster model or the real person, a grid may be projected onto it. This method was used by Information International to enter the data of actor Peter Fonda's head into their computers for the movie Futureworld (Crow 1978).

\section{TRANSFORMATIONS OF EXISTING SYNTHETIC ACTORS}

As shown in the last section, the creation of new synthetic actors is a tedious and delicate task. The situation may be improved by introducing tools to model synthetic actors. Two approaches have been introduced by Magnenat-Thalmann et al. (1989) and will be briefly reviewed in the next sections:

1. A tool for generating a new synthetic actor obtained by interpolation between two existing actors

2. A tool for modifying and editing an existing synthetic actor using local transformations and sets

\subsection{Shape Interpolation Between Human Faces}

A shape interpolation consists in generating an inbetween human face from two given human faces. The main problem of this method is that both original faces may have different numbers of facets and vertices. The technique consists in extracting profiles of both human faces from selected planes and generating two grids which correspond to the original faces. Then a correspondence is establishing between the profiles, then the correspondence between the parallel sections is found using a similar method. Now the correspondence between points is straightforward. Finally an inbetween human face is just obtained by linear interpolation. The technique has been succesfully used in the film Galaxy Sweetheart to transform th synthetc actress Marilyn into the synthetic actor Bogey.

\subsection{Introduction to local deformations}

Local deformations are probably the best for the modification and even creation of human surfaces. Several authors have proposed methods to perform limited local deformations (Barr 1984; Sederberg and Parry 1986). Numerous methods based on parametric surfaces are extensively used in CAD and CAE. As these methods deal with control points, they are not suitable for human modeling except when no resemblance with existing people is required (Nahas et al. 1987; Komatsu 1988). Second, field functions (Wyvill and Wyvill 1989) model free-form surfaces and their local deformations, but the application of this for the creation of well-known personalities seems difficult.

Allan et al. (1989) proposed a general method for manipulating polygonal meshes. They introduced a basic operation "move-vertex", which specifies a new 3D position for a specific vertex called the current vertex. Their basic operation is extended in several ways: definition of a range of influence around the current vertex, decay function over the range of influence, binding and anchoring. Other operations include stretch, grow and randomize.

\subsection{The use of sets and local deformations}

An interactive editor based on local deformations has been developed using local deformations (MagnenatThalmann et al. 1989) and the concept of sets, which are non-ordered collections of elements. Sets are used to select regions of the surface to be processed. The main point is that manipulation commands work with sets and not with objects. There are several ways of building a set:

1. The set is built from the elements belonging to a given volume: e.g. an ellipsoid volume.

2. Vertices or polygons are selected by drawing a $2 \mathrm{D}$ region using the mouse.

3. Polygons in a color range are selected.

4. The set is obtained as a result of a set operation: union, intersection and difference.

Local transformations only affect sets. Three kinds of local transformations may be applied:

- Individual transformations only affect one vertex of the current figure.

- Scaling operations move all vertices of a set according to a type of scaling and a transformation function.

- In Parallel transformations, all vertices of a set are translated according to the transformation function. 
Fig. 1 shows how new faces may be obtained from Marilyn's face.

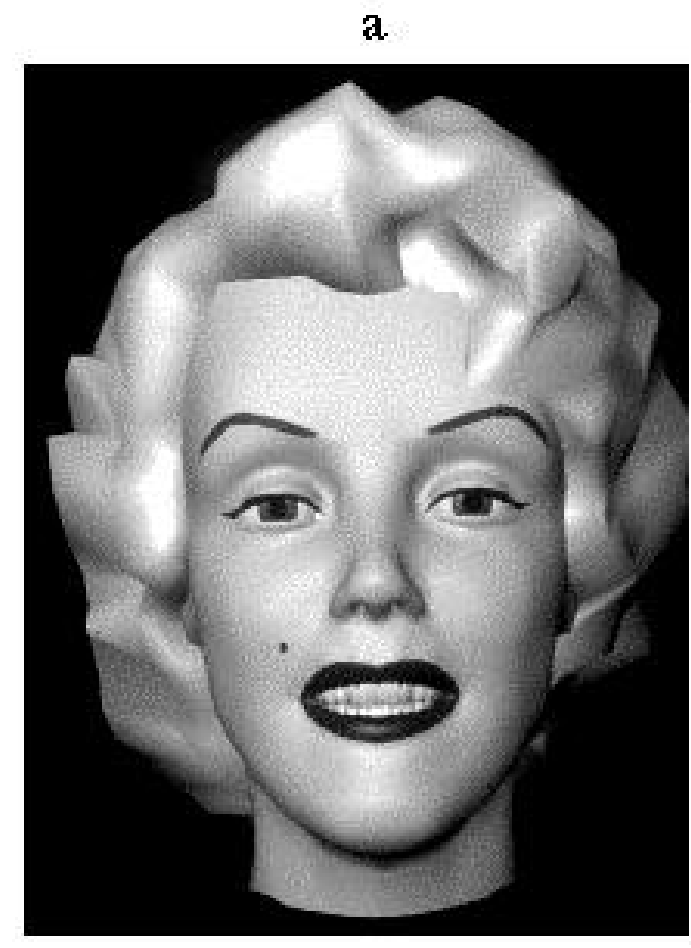

d

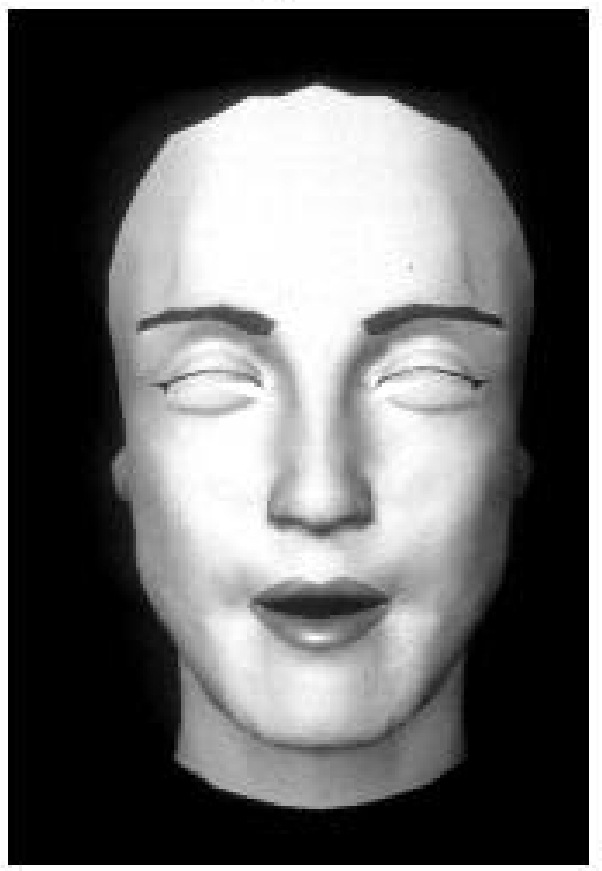

b

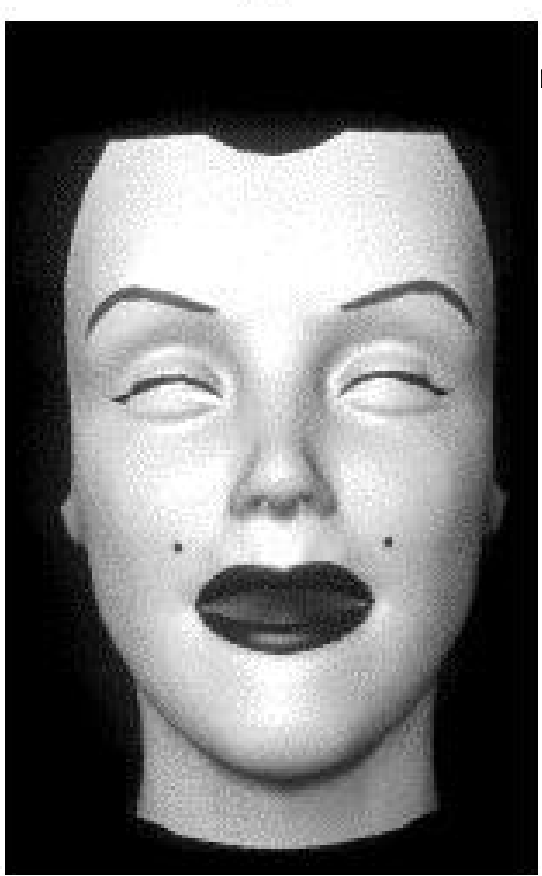

e

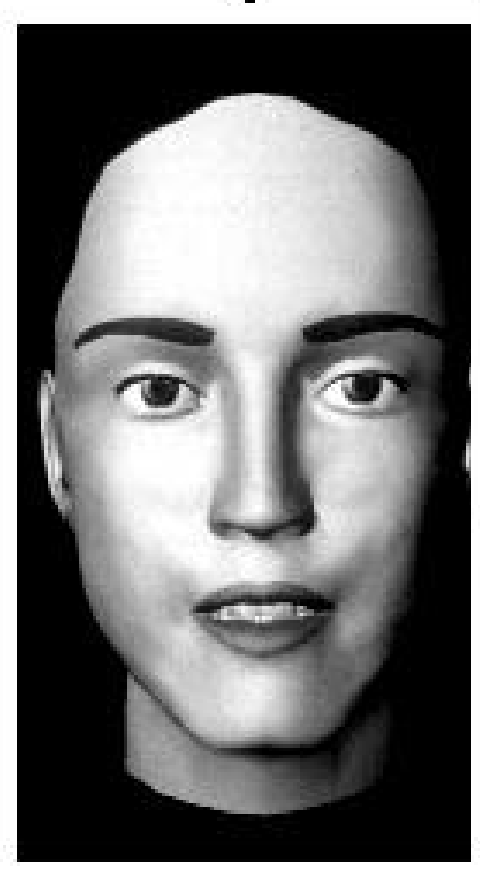

c

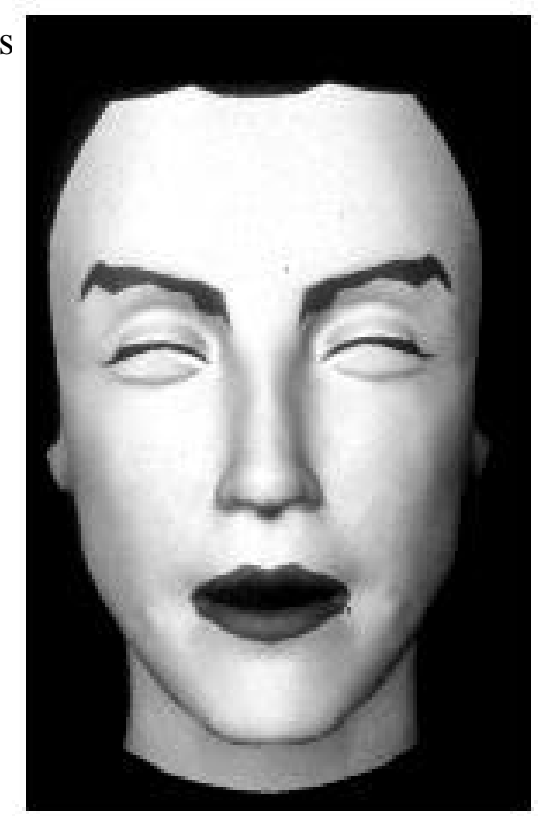

f

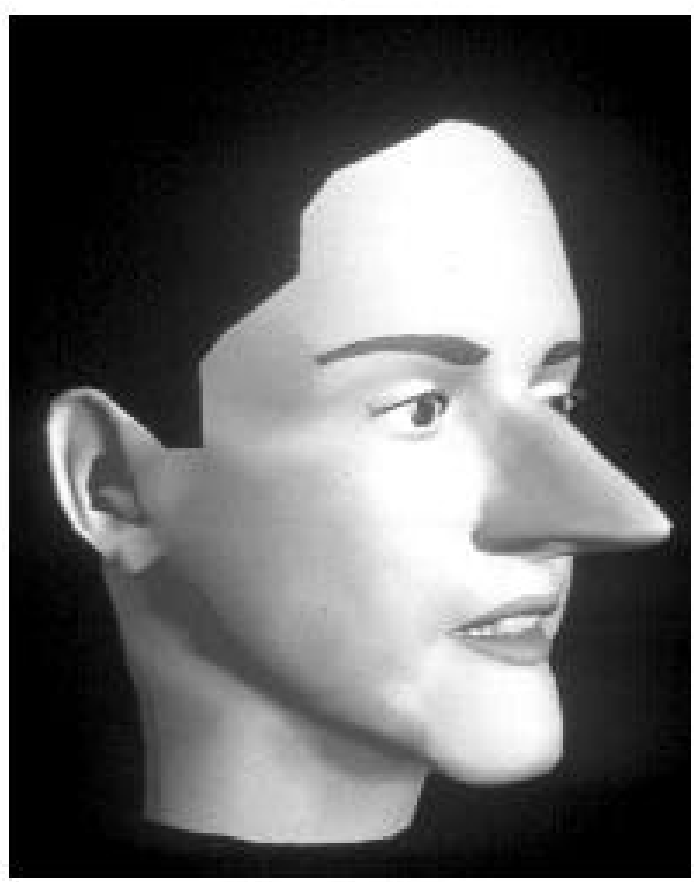

Fig.1 Transformation from Marilyn to Madonna

a) original Marilyn

b) Marilyn's face (without hair and teeth)

c) d) intermediate steps using local transformations

e) Madonna's face (without hair)

f) exaggerate face (based on Madonna's face) 


\section{THE SCULPTURE APPROACH}

Methods based on local deformations are certainly the best for modeling human shapes. However, the designer-machine interface is essential. In this section, we show how a realistic human character may be produced with a method similar to the modelling of clay, work which essentially consists of adding or eliminating parts of the material, and turning around the object when the principal form has been set up.

\subsection{Traditional sculpture}

For sculpting an object, the sculptor selects a specific material corresponding to the type of the object wished. The chosen material constrains the type of treatment. We can consider the different actions involved for different types of materials:

Adding: (materials as the clay and wax)

The sculptor sets up a wooden or metal framework with the model's size, proportions and movement and then he adds matter in order to find the preliminary shape, and establish the volume of the model. His hands are dipping into the matter in order to describe the model's form, more and more in detail.

Removing: (materials such as stone,marble, wood...)

The sculptor is looking for the model's form in the interior of a mass. The form comes after eliminating parts.

Assembling: (principally materials such as metal,but also any other heterogeneous materials coming from the industry or the nature)

In this case, the sculptor creates a new sculpture joining the elements together and giving a very different form from the beginning.

\subsection{Sculpting using computer}

The operations conducted in a traditional sculpture can be performed by computer for computer generated objects. A sculpting software (Leblanc et al. 1991) which is based on an interactive input device called the Spaceball. This allows the user to create a polygon mesh surface. When used in conjunction with a common 2-D mouse such that the Spaceball is held in one hand and the mouse in the other, full threedimensional user interaction is achieved.

The Spaceball device is used to move around the object being sculpted in order to examine it from various points of view, while the mouse carries out the picking and deformation work onto a magnifying image in order to see every small detail in real time (e.g. vertex creation, primitive selection and local surface deformations). In this way, the user not only sees the object from every angle but he can also apply and correct deformations from every angle interactively.

With this type of 3-dimensional interaction, the operations performed while sculpting an object closely resemble traditional sculpting. The major operations performed using this software include creation of primitives, selection, local surface deformations and global deformations. These are described briefly in the next sections.

\subsubsection{Creation}

Typically, the sculpting process may be initiated in two ways: by loading and altering an existing shape or by simply starting one from scratch. For example, we will use a sphere as a starting point for the head of a person and use cylinders for limbs.

We will then add or remove polygons according to the details needed and apply local deformations to alter the shape. When starting from scratch points are placed in 3D space and polygonized. However, it may be more tedious and time consuming.

\subsubsection{Selection}

To select parts of the objects, the mouse is used in conjunction with the Spaceball to quickly mark out the desired primitives in and around the object. This amounts to pressing the mouse button and sweeping the mouse cursor on the screen while moving the object with the Spaceball. All primitives (vertices, edges and 
polygons) can be selected. Mass picking may be done by moving the object away from the eye (assuming a perspective projection) and careful,minute picking may be done by bringing the object closer.

\subsubsection{Local surface deformations.}

These tools make it possible to produce local elevations or depressions on the surface and to even out unwanted bumps once the work is nearing completion.

Local deformations are applied while the Spaceball device is used to move the object and examine the progression of the deformation from different angles, mouse movements on the screen are used to produce vertex movements in 3D space from the current viewpoint.

The technique is intended to be a metaphor analogous to pinching, lifting and moving of a stretchable fabric material. Pushing the apex vertex inwards renders a believable effect of pressing a mould into clay.

\subsubsection{The global deformations}

These tools make it possible to produce global deformations on the whole object or some of the selected regions. For example, if the object has to grow in a certain direction, it can be obtained by scaling or shifting the object on the region of interest.

\section{A CASE STUDY OF DESIGN PROCESS: ELVIS}

In this section, we discuss a current project of creating a new synthetic actor Elvis. This project began by buying two books about Elvis life with several photographs. After getting familiar with the character, the design process involved breaking the human body into different logical anatomical parts.

Although Elvis' head (see Plate 2) had been modeled from a sphere, for the body we decided to do it neither from scratch, nor by digitalitalisation, but to take different parts of Marilyn's body such as legs, foot, arms as well as some other parts from Humphrey's figure such as hands and ears already available in the computer. They had been created using the 3D reconstruction from $2 \mathrm{D}$ photos, then improved using the LOCALTRANS software and we loaded them in the sculpting software as the starting point of the sculpting session.

In the modeling process, the following steps were used: extraction, composition, join, finding the preliminary figure, finishing process.

\section{Extraction}

Parts (such as arms, feet, etc.) were extracted from the other figures (see Plate 3) before adapting and joining them with the Elvis head. To do this we created another figure which contained the vertices, and corresponding polygons. In this manner we had a man's hands, a woman's torso,arms and legs as well as Elvis' original head. All these separate parts had different proportions and different colours.

\section{Composition}

This step involved the creation of one figure with positioning and assembling together the different parts, as exactly as possible according to the general proportions of the figure we want to create. Fig. 2 shows the wire-frame composition of head and torso. This also required making the figure and his parts fit on the skeleton which will serve for the animation. This implied the use of elements common to both figures to allow them to be assembled as shown in Plate 4. The topology took into consideration the contours of the head and body and the position of the points necessary for animation.

The existing skeleton constrained us to :

1.The vertebral column should be as straight as possible

2. The arms should be perpendicular to the body. 


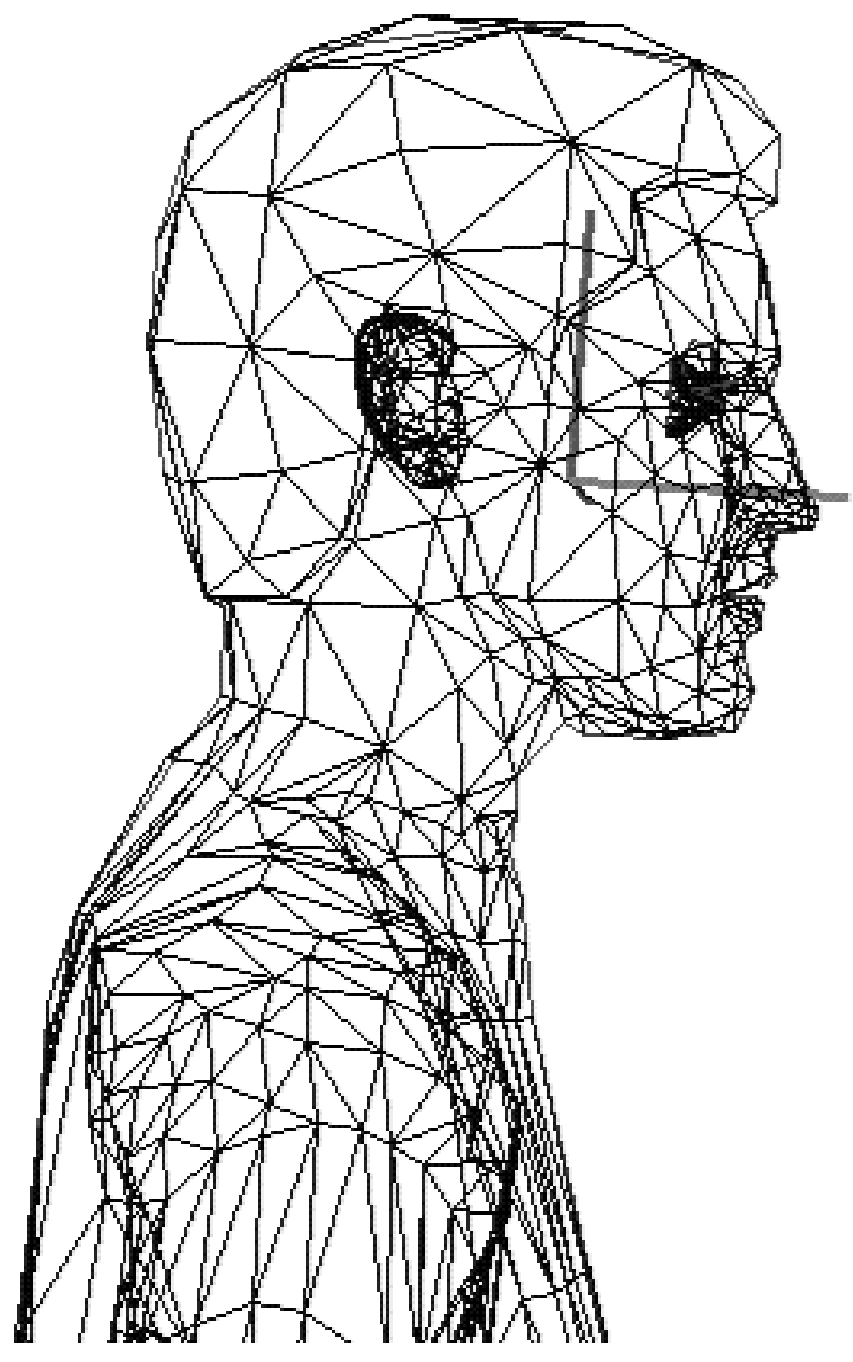

Fig.2 Composition of head and torso

Next we divide the body within a symmetry axis. Although we knew that a human face and body are not perfectly symmetric, we chose to work in the half figure because the manipulation is easier and the basic elements like facets and vertices are less. In particular, the display process is faster.

\section{Join}

In this step we had to create new polygons to join the parts, taking care to keep the continuity between the morphology of the existing figure and his new part. Plate 5 shows the process.

\section{Find the preliminary figure}

Since the different parts of the body belonged to different characters, getting the proportioned figure of the desired character required some global and local deformations to be performed. For example, Plate 6 illustrates some global deformations applied to the selected regions. These deformations composed to the feedback obtained from the document in reference for the design process. At this moment we don't lose time to correct the imperfections in detail.

To eliminate problems appeared with scaling, we then use local deformations after selecting a vertex or a groupe of vertices. In this case deforming several vertices at the same time gave a more coherent result than deforming them with the same manner one by one. 
We continue to work on the half of the figure but we frequently viewed the full figure in order to keep the whole volume in mind. In fact if we wanted to give the maximum of power and beauty we are forced to take in consideration the exterior contour .

Sometimes while working to find the preliminary shape we felt the need to give an accent to some characteristic details of the figure. At this point we would remove, exaggerate or soften different details .

\section{The finishing process}

The finishing process started when all the proportions had been found. Next we started to look at every part in detail and it was evident that this was the most delicate and difficult part of the work, which took much more time than the earlier parts. In this step we used the local surface deformations .

We divided the problems into two principal types:

1.defaults to eliminate

2.improvements wished

Examples of defaults to eliminate are details such as marks, black points, picks, etc. which had to simply be removed from the figure. The improvements were any changes required in order to make the figure acceptable.

In the construction of a certain category of figures like realistic human bodies, it is often preferable to keep certain irregularities on the surface. A very smooth skin, for example, does not necessarily guarantees a more realistic appearance. In very delicate parts like shoulders or cheeks the imperfections are visible and generally we have to get rid of them right away, but we can keep some others small irregularities in order to create a figure which seems less plastic and robotized and in order to attenuate the feeling we often feel in front of computer generated human bodies. This kind of imperfections in a realistic figure give the impression of a human figure which has not been conceived only in the "designer's" head but has really been observed from the reality.

These tools make it possible to produce local elevations or depressions on the surface and to even out unwanted bumps once the work is nearing completion.

Plate 7 shows several body's views.

\section{CONCLUSION}

As our understanding of reality and our tools become more sophisticated, we are beginning to simulate realistic environnements that even allow various degrees of physical interaction. We are able to make any character from the already existing characters in an intuitive way using natural gestures such as sculpting of different materials. This method of sculpting becomes more interesting and creative.

\section{Acknowledgements}

Sincere thanks to Prem Kalra and Russell Turner for their help in revising this text. The transformation from Marilyn to Madonna and the first Elvis' head were modelled by Ross Racine. The sculpting software has been implemented by André LeBlanc and Prem Kalra. The project has been partly sponsored by le Fonds National Suisse pour la Recherche Scientifique.

\section{References}

Allan JB, Wyvill B, Witten IA (1989) A Methodology for Direct Manipulation of Polygon Meshes, Proc. Computer Graphics International '89, Leeds, pp.451-469.

Barr AH (1984) Global and local deformations of solid primitives. Proc. SIGGRAPH '84, Computer Graphics 18(3):21-30

Crow FC (1978) Shaded Computer Graphics in the Entertainment Industry, Computer, Vol. 11, No3, pp.11-22

Komatsu, K (1988) Human skin model capable of natural shape variation, The Visual Computer, Vol.3, No5, pp.265-271 
LeBlanc A, Kalra P, Magnenat-Thalmann N, Thalmann D (1991) Sculpting With the "Ball \& Mouse" Metaphor - Proc. Graphics Interface '91, Calgary, Canada

Magnenat-Thalmann N, Thalmann D (1987) The Direction of Synthetic Actors in the Film Rendez-vous à Montréal, IEEE Computer Graphics and Applications, Vol. 7, No12

Magnenat-Thalmann N, Minh HT, de Angelis M, Thalmann D (1989) Design, Transformation and Animation of Human Faces, The Visual Computer, Vol.5, No3, pp.32-39

Magnenat Thalmann N, Thalmann D (1990), Synthetic Actors in Computer-Generated 3D Films, Springer-verlag, Tokyo

Nahas M, Huitric H, Rioux M, Domey J (1990) Proc. Computer Animation 90, Springer, Tokyo

Nahas M, Huitric H, Saintourens M (1987) Animation of a B-spline figure, The Visual Computer, Vol.3, No5,pp.272-276.

Sederberg TW, Parry SR (1986) Free-form deformation of solid geometric models. Proc. SIGGRAPH '86, Computer Graphics, Vol.20, No4, pp.151-160

Wyvill B, Wyvill G (1989) Field functions for iso-surfaces, The visual Computer, Vol.5, No3 
Plate 1: Marilyn

Plate 2. Elvis' head (preliminary version)

Plate 3: Family photo

Plate 4. Bogey's hands, Marilyn's torso and arms, Elvis' head

Plate 5. Adding polygons

Plate 6. Sequence of images showing the selected parts for global deformations

Figure 7 Several body views 
Arghyro Paouri is assistant in the Computer Graphics Lab in the University of Geneva since 1989. She had studied "Arts and Technologies" in the University of Paris VIII as well as in the "Ecole Nationale Supérieure des Beaux Arts" in Paris.

Her interests are image synthesis and computer animation.

Nadia Magnenat Thalmann is currently full Professor of Computer Science at the University of Geneva, Switzerland and Adjunct Professor at HEC Montreal, Canada. She has served on a variety of government advisory boards and program committees in Canada. She has received several awards, including the 1985 Communications Award from the Government of Quebec. In May 1987, she was nominated woman of the year in sciences by the Montreal community. Dr. Magnenat Thalmann received a BS in psychology, an MS in biochemistry, and a Ph.D in quantum chemistry and computer graphics from the University of Geneva. She has written and edited several books and research papers in image synthesis and computer animation and was codirector of the computer-generated films Dream Flight, Eglantine, Rendez-vous à Montréal, Galaxy Sweetheart, IAD and Flashback. She served as chairperson of Graphics Interface '85, CGI '88, Computer Animation '89 and Computer Animation '90.

E-mail: thalmanneuni2a.unige.ch

Daniel Thalmann is currently full Professor and Director of the Computer Graphics Laboratory at the Swiss Federal Institute of Technology in Lausanne, Switzerland. Since 1977, he was Professor at the University of Montreal and codirector of the MIRALab research laboratory. He received his diploma in nuclear physics and Ph.D in Computer Science from the University of Geneva. He is coeditor-in-chief of the Journal of Visualization and Computer Animation, member of the editorial board of the Visual Computer and cochairs the EUROGRAPHICS Working Group on Computer Simulation and Animation. Daniel Thalmann's research interests include 3D computer animation, image synthesis, and scientific visualization. He has published more than 100 papers in these areas and is coauthor of several books including: Computer Animation: Theory and Practice and Image Synthesis: Theory and Practice. He is also codirector of several computer-generated films. E-mail: thalmann@eldi.epfl.CH

The authors may be contacted at:

Computer Graphics Lab

Swiss Federal Institute of Technology

CH 1015 Lausanne

Switzerland

tel: $++41-21-693-5214$

fax: ++ 41-21-693-3909
MIRALab, CUI

University of Geneva

12 rue du Lac

CH 1207 Geneva

Switzerland

tel: ++ 41-22-787-6581

fax: ++ 41-22-735-3905 